\title{
MORE ON REVERSE TRIANGLE INEQUALITY IN INNER PRODUCT SPACES
}

\author{
A. H. ANSARI AND M. S. MOSLEHIAN
}

Received 8 February 2005 and in revised form 17 May 2005

Refining some results of Dragomir, several new reverses of the generalized triangle inequality in inner product spaces are given. Among several results, we establish some reverses for the Schwarz inequality. In particular, it is proved that if $a$ is a unit vector in a real or complex inner product space $(H ;\langle\cdot, \cdot\rangle), r, s\rangle 0, p \in(0, s], D=\{x \in H, \| r x-$ $s a \| \leq p\}, x_{1}, x_{2} \in D-\{0\}$, and $\alpha_{r, s}=\min \left\{\left(r^{2}\left\|x_{k}\right\|^{2}-p^{2}+s^{2}\right) / 2 r s\left\|x_{k}\right\|: 1 \leq k \leq 2\right\}$, then $\left(\left\|x_{1}\right\|\left\|x_{2}\right\|-\operatorname{Re}\left\langle x_{1}, x_{2}\right\rangle\right) /\left(\left\|x_{1}\right\|+\left\|x_{2}\right\|\right)^{2} \leq \alpha_{r, s}$.

\section{Introduction}

It is interesting to know under which conditions the triangle inequality went the other way in a normed space $X$; in other words, we would like to know if there is a positive constant $c$ with the property that $c \sum_{k=1}^{n}\left\|x_{k}\right\| \leq\left\|\sum_{k=1}^{n} x_{k}\right\|$ for any finite set $x_{1}, \ldots, x_{n} \in X$. Nakai and Tada [7] proved that the normed spaces with this property are precisely those of finite dimensional.

The first authors investigating reverse of the triangle inequality in inner product spaces were Diaz and Metcalf [2] by establishing the following result as an extension of an inequality given by Petrovich [8] for complex numbers.

Theorem 1.1 (Diaz-Metcalf theorem). Let a be a unit vector in an inner product space $(H ;\langle\cdot, \cdot\rangle)$. Suppose the vectors $x_{k} \in H, k \in\{1, \ldots, n\}$ satisfy

$$
0 \leq r \leq \frac{\operatorname{Re}\left\langle x_{k}, a\right\rangle}{\left\|x_{k}\right\|}, \quad k \in\{1, \ldots, n\} .
$$

Then

$$
r \sum_{k=1}^{n}\left\|x_{k}\right\| \leq\left\|\sum_{k=1}^{n} x_{k}\right\|
$$


where equality holds if and only if

$$
\sum_{k=1}^{n} x_{k}=r \sum_{k=1}^{n}\left\|x_{k}\right\| a
$$

Inequalities related to the triangle inequality are of special interest (cf. [6, Chapter XVII]). They may be applied to get interesting inequalities in complex numbers or to study vector-valued integral inequalities $[4,5]$.

Using several ideas and following the terminology of $[4,5]$, we modify or refine some results of Dragomir and ours [1] and get some new reverses of triangle inequality. Among several results, we show that if $a$ is a unit vector in a real or complex inner product $\operatorname{space}(H ;\langle\cdot, \cdot\rangle), x_{k} \in H-\{0\}, 1 \leq k \leq n, \alpha=\min \left\{\left\|x_{k}\right\|: 1 \leq k \leq n\right\}, p \in\left(0, \sqrt{\alpha^{2}+1}\right)$, $\max \left\{\left\|x_{k}-a\right\|: 1 \leq k \leq n\right\} \leq p$, and $\beta=\min \left\{\left(\left\|x_{k}\right\|^{2}-p^{2}+1\right) / 2\left\|x_{k}\right\|: 1 \leq k \leq n\right\}$, then

$$
\sum_{k=1}^{n}\left\|x_{k}\right\|-\left\|\sum_{k=1}^{n} x_{k}\right\| \leq \frac{1-\beta}{\beta} \operatorname{Re}\left\langle\sum_{k=1}^{n} x_{k}, a\right\rangle .
$$

We also examine some reverses for the celebrated Schwarz inequality. In particular, it is proved that if $a$ is a unit vector in a real or complex inner product space $(H ;\langle\cdot, \cdot\rangle), r, s\rangle 0$, $p \in(0, s], D=\{x \in H,\|r x-s a\| \leq p\}, x_{1}, x_{2} \in D-\{0\}$, and $\alpha_{r, s}=\min \left\{\left(r^{2}\left\|x_{k}\right\|^{2}-p^{2}+\right.\right.$ $\left.\left.s^{2}\right) / 2 r s\left\|x_{k}\right\|: 1 \leq k \leq 2\right\}$, then

$$
\frac{\left\|x_{1}|||| x_{2}\right\|-\operatorname{Re}\left\langle x_{1}, x_{2}\right\rangle}{\left(\left\|x_{1}\right\|+\left\|x_{2}\right\|\right)^{2}} \leq \alpha_{r, s}
$$

Throughout the paper, $(H ;\langle\cdot, \cdot\rangle)$ denotes a real or complex inner product space. We use repeatedly the Cauchy-Schwarz inequality without mentioning it. The reader is referred to $[3,9]$ for the terminology on inner product spaces.

\section{Reverse of triangle inequality}

We start this section by pointing out the following theorem of [1] which is a modification of [5, Theorem 3].

TheOREM 2.1. Let $a_{1}, \ldots, a_{m}$ be orthonormal vectors in the complex inner product space $(H ;\langle\cdot, \cdot\rangle)$. Suppose that for $1 \leq t \leq m, r_{t}, \rho_{t} \in R$, and that the vectors $x_{k} \in H, k \in\{1, \ldots, n\}$ satisfy

$$
0 \leq r_{t}^{2}\left\|x_{k}\right\| \leq \operatorname{Re}\left\langle x_{k}, r_{t} a_{t}\right\rangle, \quad 0 \leq \rho_{t}^{2}\left\|x_{k}\right\| \leq \operatorname{Im}\left\langle x_{k}, \rho_{t} a_{t}\right\rangle, \quad 1 \leq t \leq m
$$

Then

$$
\left(\sum_{t=1}^{m}\left(r_{t}^{2}+\rho_{t}^{2}\right)\right)^{1 / 2} \sum_{k=1}^{n}\left\|x_{k}\right\| \leq\left\|\sum_{k=1}^{n} x_{k}\right\|
$$


and the equality holds in (2.2) if and only if

$$
\sum_{k=1}^{n} x_{k}=\sum_{k=1}^{n}\left\|x_{k}\right\| \sum_{t=1}^{m}\left(r_{t}+i \rho_{t}\right) a_{t} .
$$

The following theorem is a strengthen of [5, Corollary 1] and a generalization of [1, Theorem 2].

Theorem 2.2. Let a be a unit vector in the complex inner product space $(H ;\langle\cdot, \cdot\rangle)$. Suppose that the vectors $x_{k} \in H-\{0\}, k \in\{1, \ldots, n\}$ satisfy

$$
\max \left\{\left\|r x_{k}-s a\right\|: 1 \leq k \leq n\right\} \leq p, \quad \max \left\{\left\|r^{\prime} x_{k}-i s^{\prime} a\right\|: 1 \leq k \leq n\right\} \leq q,
$$

where $r, r^{\prime}, s, s^{\prime}>0$ and

$$
\begin{gathered}
p \leq\left((r \alpha)^{2}+s^{2}\right)^{1 / 2}, \quad q \leq\left(\left(r^{\prime} \alpha\right)^{2}+s^{\prime 2}\right)^{1 / 2}, \\
\alpha=\min \left\{\left\|x_{k}\right\|: 1 \leq k \leq n\right\} .
\end{gathered}
$$

Let

$$
\begin{aligned}
\alpha_{r, s} & =\min \left\{\frac{r^{2}\left\|x_{k}\right\|^{2}-p^{2}+s^{2}}{2 r s\left\|x_{k}\right\|}: 1 \leq k \leq n\right\}, \\
\beta_{r^{\prime}, s^{\prime}} & =\min \left\{\frac{r^{\prime 2}\left\|x_{k}\right\|^{2}-q^{2}+s^{\prime 2}}{2 r^{\prime} s^{\prime}\left\|x_{k}\right\|}: 1 \leq k \leq n\right\} .
\end{aligned}
$$

Then

$$
\left(\alpha_{r, s}^{2}+\beta_{r^{\prime}, s^{\prime}}^{2}\right)^{1 / 2} \sum_{k=1}^{n}\left\|x_{k}\right\| \leq\left\|\sum_{k=1}^{n} x_{k}\right\|
$$

and the equality holds if and only if

$$
\sum_{k=1}^{n} x_{k}=\left(\alpha_{r, s}+i \beta_{r^{\prime}, s^{\prime}}\right) \sum_{k=1}^{n}\left\|x_{k}\right\| a .
$$

Proof. From the first inequality above, we infer that

$$
\begin{gathered}
\left\langle r x_{k}-s a, r x_{k}-s a\right\rangle \leq p^{2}, \\
r^{2}\left\|x_{k}\right\|^{2}+s^{2}-p^{2} \leq 2 \operatorname{Re}\left\langle r x_{k}, s a\right\rangle .
\end{gathered}
$$

Then

$$
\frac{r^{2}\left\|x_{k}\right\|^{2}+s^{2}-p^{2}}{2 r s\left\|x_{k}\right\|}\left\|x_{k}\right\| \leq \operatorname{Re}\left\langle x_{k}, a\right\rangle .
$$

Similarly,

$$
\frac{r^{\prime 2}\left\|x_{k}\right\|^{2}-q^{2}+s^{\prime 2}}{2 r^{\prime} s^{\prime}\left\|x_{k}\right\|}\left\|x_{k}\right\| \leq \operatorname{Im}\left\langle x_{k}, a\right\rangle,
$$


2886 Reverse triangle inequality

consequently,

$$
\begin{aligned}
\alpha_{r, s}\left\|x_{k}\right\| & \leq \operatorname{Re}\left\langle x_{k}, a\right\rangle, \\
\beta_{r^{\prime}, s^{\prime}}\left\|x_{k}\right\| & \leq \operatorname{Im}\left\langle x_{k}, a\right\rangle .
\end{aligned}
$$

Applying Theorem 2.1 for $m=1, r_{1}=\alpha_{r, s}$, and $\rho_{1}=\beta_{r^{\prime}, s^{\prime}}$, we deduce the desired inequality.

The next result is an extension of [1, Corollary 3$]$.

Corollary 2.3. Let a be a unit vector in the complex inner product space $(H ;\langle\cdot, \cdot\rangle)$. Suppose that $x_{k} \in H, k \in\{1, \ldots, n\}, \max \left\{\left\|r x_{k}-s a\right\|: 1 \leq k \leq n\right\} \leq r, \max \left\{\left\|r x_{k}-i s a\right\|: 1 \leq\right.$ $k \leq n\} \leq s$, where $r>0, s>0$, and $\alpha=\min \left\{\left\|x_{k}\right\|: 1 \leq k \leq n\right\}$. Then

$$
\frac{r \alpha}{s \sqrt{2}} \sum_{k=1}^{n}\left\|x_{k}\right\| \leq\left\|\sum_{k=1}^{n} x_{k}\right\| .
$$

The equality holds if and only if

$$
\sum_{k=1}^{n} x_{k}=r \alpha \frac{(1+i)}{2 s} \sum_{k=1}^{n}\left\|x_{k}\right\| a
$$

Proof. Apply Theorem 2.2 with $r=r^{\prime}, s=s^{\prime}, p=r, q=s$. Note that $\alpha_{r, s}=r \alpha / 2 s=\beta_{r^{\prime}, s^{\prime}}$.

Theorem 2.4. Let a be a unit vector in $(H ;\langle\cdot, \cdot\rangle)$. Suppose that the vectors $x_{k} \in H, k \in$ $\{1, \ldots, n\}$ satisfy

$$
\max \left\{\left\|r x_{k}-s a\right\|: 1 \leq k \leq n\right\} \leq p<\left((r \alpha)^{2}+s^{2}\right)^{1 / 2},
$$

where $r>0, s>0$ and

$$
\alpha=\min _{1 \leq k \leq n}\left\|x_{k}\right\|
$$

Let

$$
\alpha_{r, s}=\min \left\{\frac{r^{2}\left\|x_{k}\right\|^{2}-p^{2}+s^{2}}{2 r s\left\|x_{k}\right\|}: 1 \leq k \leq n\right\} .
$$

Then

$$
\alpha_{r, s} \sum_{k=1}^{n}\left\|x_{k}\right\| \leq\left\|\sum_{k=1}^{n} x_{k}\right\| .
$$

Moreover, the equality holds if and only if

$$
\sum_{k=1}^{n} x_{k}=\alpha_{r, s} \sum_{k=1}^{n}\left\|x_{k}\right\| a
$$


Proof. Proof is similar to that of Theorem 2.2 in which we use Theorem 2.1 with $m=1$, $\rho_{1}=0$.

Theorem 2.5. Let a be a unit vector in $(H ;\langle\cdot, \cdot\rangle)$. Suppose that $r, s\rangle 0$, and vectors $x_{k} \in$ $H-\{0\}, k \in\{1, \ldots, n\}$ satisfy

$$
\sum_{k=1}^{n} x_{k}=0
$$

Then

$$
\sqrt{r^{2} \alpha^{2}+s^{2}} \leq \max \left\{\left\|r x_{k}-s a\right\|: 1 \leq n\right\}
$$

where

$$
\alpha=\min _{1 \leq k \leq n}\left\|x_{k}\right\|
$$

Proof. Let $p=\max \left\{\left\|r x_{k}-s a\right\|: 1 \leq k \leq n\right\}$. If $p<\sqrt{r^{2} \alpha^{2}+s^{2}}$, then, using Theorem 2.4, we get

$$
\alpha_{r, s} \sum_{k=1}^{n}\left\|x_{k}\right\| \leq\left\|\sum_{k=1}^{n} x_{k}\right\|=0
$$

Hence $\alpha_{r, s}=0$. On the other hand, $\left(p^{2}-s^{2}\right) / r^{2}<\alpha^{2}$, so

$$
\alpha_{r, s}=\min \left\{\frac{r^{2}\left\|x_{k}\right\|^{2}-p^{2}+s^{2}}{2 r s\left\|x_{k}\right\|}: 1 \leq k \leq n\right\}>0
$$

holds a contradiction.

THeORem 2.6. Let $a_{1}, \ldots, a_{m}$ be orthonormal vectors in the complex inner product space $(H ;\langle\cdot, \cdot\rangle), M_{t} \geq m_{t}>0, L_{t} \geq \ell_{t}>0,1 \leq t \leq m$, and $x_{k} \in H-\{0\}, k \in\{1, \ldots, n\}$ such that

$$
\operatorname{Re}\left\langle M_{t} a_{t}-x_{k}, x_{k}-m_{t} a_{t}\right\rangle \geq 0, \quad \operatorname{Re}\left\langle L_{t} i a_{t}-x_{k}, x_{k}-\ell_{t} i a_{t}\right\rangle \geq 0,
$$

or equivalently

$$
\left\|x_{k}-\frac{m_{t}+M_{t}}{2} a_{t}\right\| \leq \frac{M_{t}-m_{t}}{2}, \quad\left\|x_{k}-\frac{L_{t}+\ell_{t}}{2} i a_{t}\right\| \leq \frac{L_{t}-\ell_{t}}{2},
$$

for all $1 \leq k \leq n$ and $1 \leq t \leq m$. Let

$$
\begin{aligned}
\alpha_{m_{t}, M_{t}} & =\min \left\{\frac{\left\|x_{k}\right\|^{2}+m_{t} M_{t}}{\left(m_{t}+M_{t}\right)\left\|x_{k}\right\|}: 1 \leq k \leq n\right\}, \quad 1 \leq t \leq m, \\
\alpha_{\ell_{t}, L_{t}} & =\min \left\{\frac{\left\|x_{k}\right\|^{2}+\ell_{t} L_{t}}{\left(m_{t}+M_{t}\right)\left\|x_{k}\right\|}: 1 \leq k \leq n\right\}, \quad 1 \leq t \leq m,
\end{aligned}
$$


2888 Reverse triangle inequality

then

$$
\left(\sum_{t=1}^{m} \alpha_{m_{t}, M_{t}}^{2}+\alpha_{\ell_{t}, L_{t}}^{2}\right)^{1 / 2} \sum_{k=1}^{n}\left\|x_{k}\right\| \leq\left\|\sum_{k=1}^{n} x_{k}\right\| .
$$

The equality holds if and only if

$$
\sum_{k=1}^{n} x_{k}=\left(\sum_{k=1}^{n}\left\|x_{k}\right\|\right) \sum_{t=1}^{m}\left(\alpha_{m_{t}, M_{t}}+i \alpha_{\ell_{t}, L_{t}}\right) a_{t} .
$$

Proof. Given $1 \leq t \leq m$ and all $1 \leq k \leq n$, it follows from $\left\|x_{k}-\left(\left(m_{t}+M_{t}\right) / 2\right) a_{t}\right\| \leq\left(M_{t}-\right.$ $\left.m_{t}\right) / 2$ that

$$
\left\|x_{k}\right\|^{2}+m_{t} M_{t} \leq\left(m_{t}+M_{t}\right) \operatorname{Re}\left\langle x_{k}, a_{t}\right\rangle
$$

Then

$$
\frac{\left\|x_{k}\right\|^{2}+m_{t} M_{t}}{\left(m_{t}+M_{t}\right)\left\|x_{k}\right\|}\left\|x_{k}\right\| \leq \operatorname{Re}\left\langle x_{k}, a_{t}\right\rangle
$$

and so

$$
\alpha_{m_{t}, M_{t}}\left\|x_{k}\right\| \leq \operatorname{Re}\left\langle x_{k}, a_{t}\right\rangle
$$

Similarly, from the second inequality, we deduce that

$$
\alpha_{\ell_{t}, L_{t}}\left\|x_{k}\right\| \leq \operatorname{Im}\left\langle x_{k}, a_{t}\right\rangle
$$

Applying Theorem 2.5 for $r_{t}=\alpha_{m_{t}, M_{t}}$ and $\rho_{t}=\alpha_{\ell_{t}, L_{t}}$, we obtain the required inequality.

We will need [4, Theorem 7]. We mention it for the sake of completeness.

Theorem 2.7. Let a be a unit vector in $(H ;\langle\cdot, \cdot\rangle)$, and $x_{k} \in H-\{0\}, k \in\{1, \ldots, n\}$. If $r_{k} \geq 0, k \in\{1, \ldots, n\}$ such that

$$
\left\|x_{k}\right\|-\operatorname{Re}\left\langle x_{k}, a\right\rangle \leq r_{k}
$$

then

$$
\sum_{k=1}^{n}\left\|x_{k}\right\|-\left\|\sum_{k=1}^{n} x_{k}\right\| \leq \sum_{k=1}^{n} r_{k}
$$

The equality holds if and only if

$$
\begin{gathered}
\sum_{k=1}^{n}\left\|x_{k}\right\| \geq \sum_{k=1}^{n} r_{k}, \\
\sum_{k=1}^{n} x_{k}=\left(\sum_{k=1}^{n}\left\|x_{k}\right\|-\sum_{k=1}^{n} r_{k}\right) a .
\end{gathered}
$$


Theorem 2.8. Let a be a unit vector in $(H ;\langle\cdot, \cdot\rangle)$, and $x_{k} \in H-\{0\}, k \in\{1, \ldots, n\}$. Let

$$
\begin{gathered}
\alpha=\min \left\{\left\|x_{k}\right\|: 1 \leq k \leq n\right\}, \quad p \in\left(0, \sqrt{\alpha^{2}+1}\right), \quad \max \left\{\left\|x_{k}-a\right\|: 1 \leq k \leq n\right\} \leq p, \\
\beta=\min \left\{\frac{\left\|x_{k}\right\|^{2}-p^{2}+1}{2\left\|x_{k}\right\|}: 1 \leq k \leq n\right\} .
\end{gathered}
$$

Then

$$
\sum_{k=1}^{n}\left\|x_{k}\right\|-\left\|\sum_{k=1}^{n} x_{k}\right\| \leq \frac{1-\beta}{\beta} \operatorname{Re}\left\langle\sum_{k=1}^{n} x_{k}, a\right\rangle .
$$

The equality holds if and only if

$$
\begin{gathered}
\sum_{k=1}^{n}\left\|x_{k}\right\| \geq \frac{1-\beta}{\beta} \operatorname{Re}\left\langle\sum_{k=1}^{n} x_{k}, a\right\rangle, \\
\sum_{k=1}^{n} x_{k}=\left(\sum_{k=1}^{n}\left\|x_{k}\right\|-\frac{1-\beta}{\beta} \operatorname{Re}\left\langle\sum_{k=1}^{n} x_{k}, a\right\rangle\right) a .
\end{gathered}
$$

Proof. Since $\max \left\{\left\|x_{k}-a\right\|: 1 \leq k \leq n\right\} \leq p$, we have

$$
\begin{gathered}
\left\langle x_{k}-a, x_{k}-a\right\rangle \leq p^{2}, \quad\left\|x_{k}\right\|^{2}+1-p^{2} \leq 2 \operatorname{Re}\left\langle x_{k}, a\right\rangle, \\
\frac{\left\|x_{k}\right\|^{2}-p^{2}+1}{2\left\|x_{k}\right\|}\left\|x_{k}\right\| \leq \operatorname{Re}\left\langle x_{k}, a\right\rangle, \quad \beta\left\|x_{k}\right\| \leq \operatorname{Re}\left\langle x_{k}, a\right\rangle, \\
\left\|x_{k}\right\| \leq \frac{1}{\beta} \operatorname{Re}\left\langle x_{k}, a\right\rangle,
\end{gathered}
$$

for all $k \in\{1, \ldots, n\}$. Then

$$
\left\|x_{k}\right\|-\operatorname{Re}\left\langle x_{k}, a\right\rangle \leq \frac{1-\beta}{\beta} \operatorname{Re}\left\langle x_{k}, a\right\rangle, \quad k \in\{1, \ldots, n\} .
$$

Applying Theorem 2.7 for $r_{k}=((1-\beta) / \beta) \operatorname{Re}\left\langle x_{k}, a\right\rangle, k \in\{1, \ldots, n\}$, we deduce the desired inequality.

As a corollary, we obtain a result similar to [4, Theorem 9].

Corollary 2.9. Let a be a unit vector in $(H ;\langle\cdot, \cdot\rangle)$, and $x_{k} \in H-\{0\}, k \in\{1, \ldots, n\}$. Let

$$
\begin{gathered}
\max \left\{\left\|x_{k}-a\right\|: 1 \leq k \leq n\right\} \leq 1, \\
\alpha=\min \left\{\left\|x_{k}\right\|: 1 \leq k \leq n\right\} .
\end{gathered}
$$

Then

$$
\sum_{k=1}^{n}\left\|x_{k}\right\|-\left\|\sum_{k=1}^{n} x_{k}\right\| \leq \frac{2-\alpha}{\alpha} \operatorname{Re}\left\langle\sum_{k=1}^{n} x_{k}, a\right\rangle .
$$


2890 Reverse triangle inequality

The equality holds if and only if

$$
\begin{gathered}
\sum_{k=1}^{n}\left\|x_{k}\right\| \geq \frac{2-\alpha}{\alpha} \operatorname{Re}\left\langle\sum_{k=1}^{n} x_{k}, a\right\rangle, \\
\sum_{k=1}^{n} x_{k}=\left(\sum_{k=1}^{n}\left\|x_{k}\right\|-\frac{2-\alpha}{\alpha} \operatorname{Re}\left\langle\sum_{k=1}^{n} x_{k}, a\right\rangle\right) a .
\end{gathered}
$$

Proof. Apply Theorem 2.8 with $\beta=\alpha / 2$.

Theorem 2.10. Let a be a unit vector in $(H ;\langle\cdot, \cdot\rangle), M \geq m>0$, and $x_{k} \in H-\{0\}, k \in$ $\{1, \ldots, n\}$ such that

$$
\operatorname{Re}\left\langle M a-x_{k}, x_{k}-m a\right\rangle \geq 0
$$

or equivalently

$$
\left\|x_{k}-\frac{m+M}{2} a\right\| \leq \frac{M-m}{2}
$$

Let

$$
\alpha_{m, M}=\min \left\{\frac{\left\|x_{k}\right\|^{2}+m M}{(m+M)\left\|x_{k}\right\|}: 1 \leq k \leq n\right\} .
$$

Then

$$
\sum_{k=1}^{n}\left\|x_{k}\right\|-\left\|\sum_{k=1}^{n} x_{k}\right\| \leq \frac{1-\alpha_{m, M}}{\alpha_{m, M}} \operatorname{Re}\left\langle\sum_{k=1}^{n} x_{k}, a\right\rangle .
$$

The equality holds if and only if

$$
\begin{gathered}
\sum_{k=1}^{n}\left\|x_{k}\right\| \geq \frac{1-\alpha_{m, M}}{\alpha_{m, M}} \operatorname{Re}\left\langle\sum_{k=1}^{n} x_{k}, a\right\rangle, \\
\sum_{k=1}^{n} x_{k}=\left(\sum_{k=1}^{n}\left\|x_{k}\right\|-\frac{1-\alpha_{m, M}}{\alpha_{m, M}} \operatorname{Re}\left\langle\sum_{k=1}^{n} x_{k}, a\right\rangle\right) a .
\end{gathered}
$$

Proof. For each $1 \leq k \leq n$, it follows from the inequality

$$
\left\|x_{k}-\frac{m+M}{2} a\right\| \leq \frac{M-m}{2}
$$

that

$$
\left\langle x_{k}-\frac{m+M}{2} a, x_{k}-\frac{m+M}{2} a\right\rangle \leq\left(\frac{M-m}{2}\right)^{2} .
$$

Hence

$$
\left\|x_{k}\right\|^{2}+m M \leq(m+M) \operatorname{Re}\left\langle x_{k}, a\right\rangle .
$$


So that

$$
\alpha_{m, M}\left\|x_{k}\right\| \leq \operatorname{Re}\left\langle x_{k}, a\right\rangle
$$

consequently

$$
\left\|x_{k}\right\|-\operatorname{Re}\left\langle x_{k}, a\right\rangle \leq \frac{1-\alpha_{m, M}}{\alpha_{m, M}} \operatorname{Re}\left\langle x_{k}, a\right\rangle .
$$

Now apply Theorem 2.7 for $r_{k}=\left(\left(1-\alpha_{m, M}\right) / \alpha_{m, M}\right) \operatorname{Re}\left\langle x_{k}, a\right\rangle, k \in\{1, \ldots, n\}$.

\section{Reverses of Schwarz inequality}

In this section, we provide some reverses of the Schwarz inequality. The first theorem is an extension of [4, Proposition 5.1].

Theorem 3.1. Let a be a unit vector in $(H ;\langle\cdot, \cdot\rangle)$. Suppose that $r, s\rangle 0, p \in(0, s]$, and

$$
D=\{x \in H,\|r x-s a\| \leq p\} .
$$

If $0 \neq x_{1} \in D, 0 \neq x_{2} \in D$, then

$$
\frac{\left\|x_{1}\right\|\left\|x_{2}\right\|-\operatorname{Re}\left\langle x_{1}, x_{2}\right\rangle}{\left(\left\|x_{1}\right\|+\left\|x_{2}\right\|\right)^{2}} \leq \frac{1}{2}\left(1-\left(\frac{r^{2}\left\|x_{1}\right\|^{2}-p^{2}+s^{2}}{2 r s\left\|x_{1}\right\|}\right)^{2}\right)
$$

or

$$
\frac{\left\|x_{1}\right\|\left\|x_{2}\right\|-\operatorname{Re}\left\langle x_{1}, x_{2}\right\rangle}{\left(\left\|x_{1}\right\|+\left\|x_{2}\right\|\right)^{2}} \leq \frac{1}{2}\left(1-\left(\frac{r^{2}\left\|x_{2}\right\|^{2}-p^{2}+s^{2}}{2 r s|| x_{2} \|}\right)^{2}\right) .
$$

Proof. Put $\alpha_{r, s}=\min \left\{\left(r^{2}\left\|x_{k}\right\|^{2}-p^{2}+s^{2}\right) / 2 r s\left\|x_{k}\right\|: 1 \leq k \leq 2\right\}$. By Theorem 2.4, we obtain

$$
\alpha_{r, s}\left(\left\|x_{1}\right\|+\left\|x_{2}\right\|\right) \leq\left\|x_{1}+x_{2}\right\|
$$

Then

$$
\alpha_{r, s}^{2}\left(\left\|x_{1}\right\|^{2}+2\left\|x_{1}\right\|\left\|x_{2}\right\|+\left\|x_{2}\right\|^{2}\right) \leq\left\|x_{1}\right\|^{2}+2 \operatorname{Re}\left\langle x_{1}, x_{2}\right\rangle+\left\|x_{2}\right\|^{2}
$$

Set $\alpha_{r, s}^{2}=1-t^{2}$. Then

$$
\frac{\left\|x_{1}\right\||| x_{2} \|-\operatorname{Re}\left\langle x_{1}, x_{2}\right\rangle}{\left(\left\|x_{1}\right\|+\left\|x_{2}\right\|\right)^{2}} \leq \frac{1}{2} t^{2}
$$

namely,

$$
\frac{\left\|x_{1}\right\||| x_{2} \|-\operatorname{Re}\left\langle x_{1}, x_{2}\right\rangle}{\left(\left\|x_{1}\right\|+\left\|x_{2}\right\|\right)^{2}} \leq \frac{1}{2}\left(1-\alpha_{r, s}^{2}\right) .
$$


Corollary 3.2. Let a be a unit vector in $(H ;\langle\cdot, \cdot\rangle)$. Suppose that $r, s\rangle 0$ and

$$
D=\{x \in H,\|r x-s a\| \leq s\} .
$$

If $x, y \in D$ and $0<\|x\|<\|y\|$, then

$$
\frac{\|x\|\|y\|-\operatorname{Re}\langle x, y\rangle}{(\|x\|+\|y\|)^{2}} \leq \frac{1}{2}\left(1-\left(\frac{r\|x\|}{2 s}\right)^{2}\right) .
$$

Proof. In the notation of the proof of Theorem 3.1, we get from $p=s, x_{1}=x, x_{2}=y$ that $\alpha_{r, s}=r\|x\| / 2 s$. Now apply Theorem 3.1.

Corollary 3.3. Let a be a unit vector in $(H ;\langle\cdot, \cdot\rangle), M \geq m\rangle 0$, and $x_{k} \in H-\{0\}, k=1,2$ such that

$$
\operatorname{Re}\left\langle M a-x_{k}, x_{k}-m a\right\rangle \geq 0
$$

or equivalently

$$
\left\|x_{k}-\frac{m+M}{2} a\right\| \leq \frac{M-m}{2}
$$

Then

$$
\frac{\left\|x_{1}\right\|\left\|x_{2}\right\|-\operatorname{Re}\left\langle x_{1}, x_{2}\right\rangle}{\left(\left\|x_{1}\right\|+\left\|x_{2}\right\|\right)^{2}} \leq \frac{1}{2}\left(1-\left(\frac{\left\|x_{1}\right\|^{2}+m M}{(m+M)\left\|x_{1}\right\|}\right)^{2}\right)
$$

or

$$
\frac{\left\|x_{1}\right\||| x_{2} \|-\operatorname{Re}\left\langle x_{1}, x_{2}\right\rangle}{\left(\left\|x_{1}\right\|+\| x_{2} \mid\right)^{2}} \leq \frac{1}{2}\left(1-\left(\frac{\left\|x_{2}\right\|^{2}+m M}{(m+M)|| x_{2} \|}\right)^{2}\right) .
$$

Proof. Put $r=1, s=(m+M) / 2, p=(M-m) / 2, x=x_{1}$, and $y=x_{2}$ in Theorem 3.1.

\section{Acknowledgment}

The authors would like to thank the referees for their valuable suggestions.

\section{References}

[1] A. H. Ansari and M. S. Moslehian, Refinements of reverse triangle inequalities in inner product spaces, JIPAM. J. Inequal. Pure Appl. Math. 6 (2005), no. 3, article 64.

[2] J. B. Diaz and F. T. Metcalf, A complementary triangle inequality in Hilbert and Banach spaces, Proc. Amer. Math. Soc. 17 (1966), no. 1, 88-97.

[3] S. S. Dragomir, Discrete Inequalities of the Cauchy-Bunyakovsky-Schwarz Type, Nova Science, New York, 2004.

[4] - Reverses of the triangle inequality in inner product spaces, Aust. J. Math. Anal. Appl. 1 (2004), no. 2, 1-14, article 7.

[5] - Some reverses of the generalised triangle inequality in complex inner product spaces, Linear Algebra Appl. 402 (2005), 245-254. 
[6] D. S. Mitrinović, J. E. Pečarić, and A. M. Fink, Classical and New Inequalities in Analysis, Mathematics and Its Applications (East European Series), vol. 61, Kluwer Academic, Dordrecht, 1993.

[7] M. Nakai and T. Tada, The reverse triangle inequality in normed spaces, New Zealand J. Math. 25 (1996), no. 2, 181-193.

[8] M. Petrovich, Module d'une somme, L’Ensignement Mathématique 19 (1917), no. 1/2, 53-56 (French).

[9] Th. M. Rassias (ed.), Inner Product Spaces and Applications, Pitman Research Notes in Mathematics Series, vol. 376, Longman, Harlow, 1997.

A. H. Ansari: Department of Mathematics, Ferdowsi University, P.O. Box 1159, Mashhad 91775, Iran

E-mail address: arsalan_h_a@yahoo.com

M. S. Moslehian: Department of Mathematics, Ferdowsi University, P.O. Box 1159, Mashhad 91775, Iran

E-mail address: moslehian@ferdowsi.um.ac.ir 


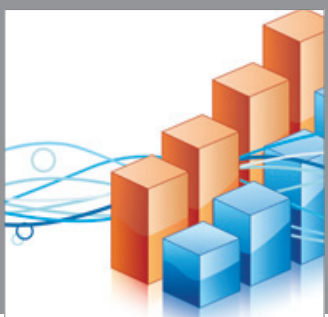

Advances in

Operations Research

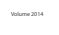

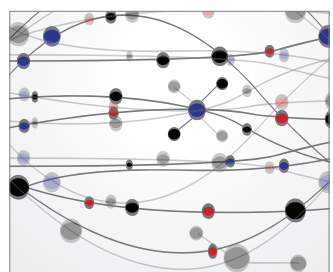

\section{The Scientific} World Journal
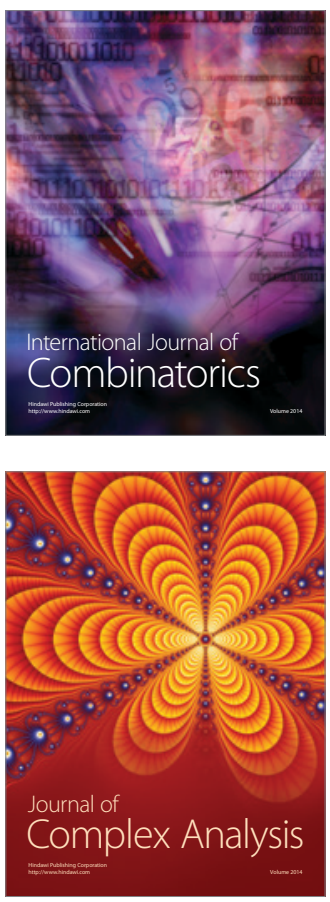

International Journal of

Mathematics and

Mathematical

Sciences
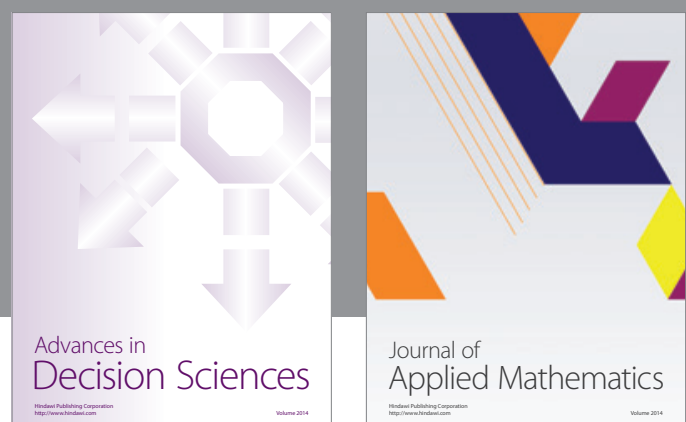

Journal of

Applied Mathematics
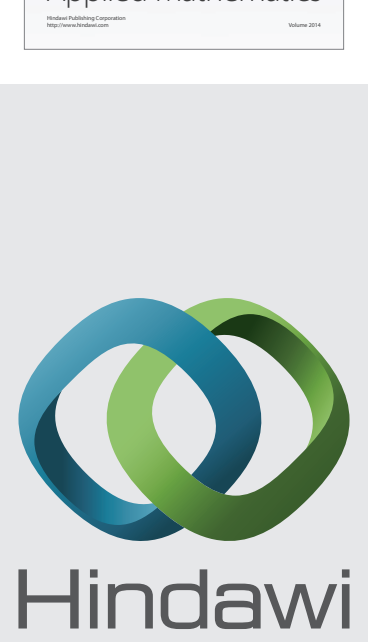

Submit your manuscripts at http://www.hindawi.com
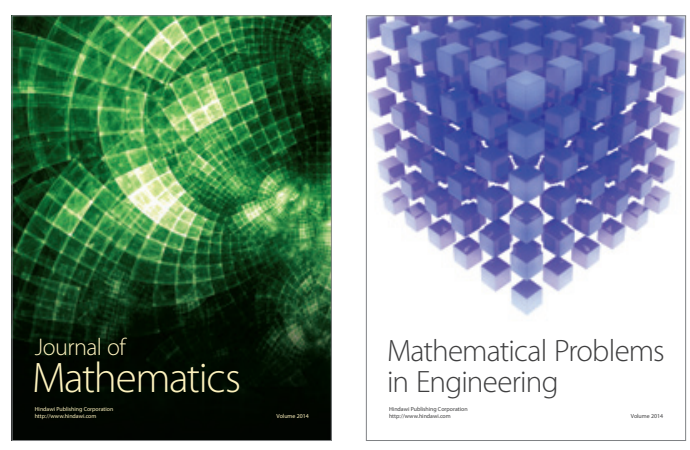

Mathematical Problems in Engineering
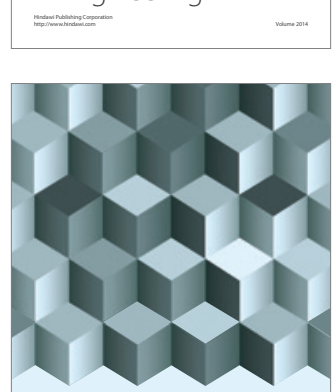

Journal of

Function Spaces
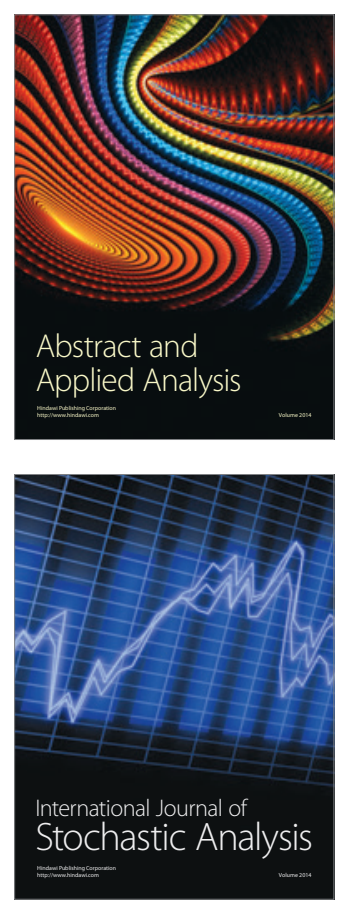

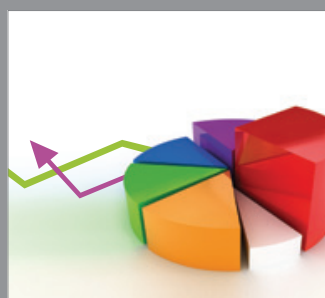

ournal of

Probability and Statistics

Promensencen
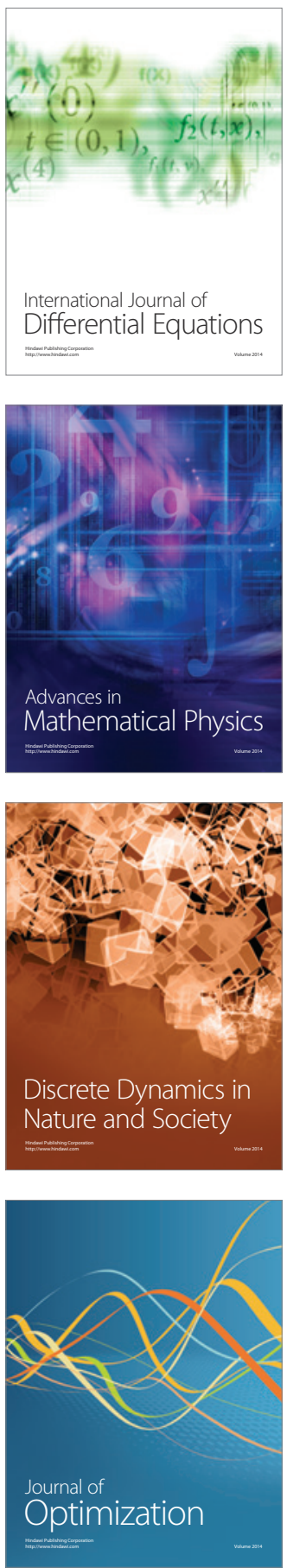